\title{
TMS of the anterior intraparietal area selectively modulates orientation change detection during action preparation
}

\author{
T. P. Gutteling, ${ }^{1,2}$ S. Y. Park, ${ }^{1}$ J. L. Kenemans, ${ }^{3}$ and S. F. W. Neggers ${ }^{1}$ \\ ${ }^{1}$ Department of Psychiatry, Rudolf Magnus Institute of Neuroscience, University Medical Center Utrecht, Utrecht, \\ The Netherlands; ${ }^{2}$ Radboud University Nijmegen, Donders Institute for Brain, Cognition and Behaviour, Nijmegen, \\ The Netherlands; and ${ }^{3}$ Department of Experimental Psychology and Psychopharmacology, Utrecht University, \\ Utrecht, The Netherlands
}

Submitted 20 July 2012; accepted in final form 10 April 2013

\begin{abstract}
Gutteling TP, Park SY, Kenemans JL, Neggers SF. TMS of the anterior intraparietal area selectively modulates orientation change detection during action preparation. J Neurophysiol 110: 33-41, 2013. First published April 17, 2013; doi:10.1152/jn.00622.2012.— Perception of relevant visual object features can be modulated by the preparation of an action toward it ("action-modulated perception"). For instance, the perception of the orientation of a book can be enhanced when preparing to grasp it (but not when pointing to it). However, the underlying neuronal mechanisms are poorly understood. We argue that brain areas controlling arm movements are involved in establishing this effect through top-down feedback to early visual areas, similar to the neuronal mechanisms linking visual attention and eye movements. To investigate this involvement, we applied transcranial magnetic stimulation to a grasping motor area, the left anterior intraparietal sulcus (aIPS), during grasping or pointing preparation. Concurrently, an orientation change detection task was performed. As a control area, the vertex was stimulated. We found that stimulation of aIPS selectively modulates orientation sensitivity during action preparation compared with control stimulation (vertex), negating the increased orientation sensitivity with grasping preparation over pointing preparation. We argue that aIPS is a critical part of the mechanism underlying perceptual modulations during action preparation. The present results and recent literature suggest that this action-modulated perception for hand movements is implemented through a cortical feedback connection between aIPS and early visual areas.
\end{abstract}

attention; feedback; grasping; motor preparation; perception

TO EXECUTE OUR ACTIONS in daily life successfully, it is beneficial to focus on specific features of the incoming visual information. Picking up a pen from the table might be more successful when one is focused on its orientation rather than its color. Obviously, it is advantageous to induce such selective perception automatically before the actual execution of the action. Initial evidence toward such an action-modulated perception was provided in a study by Craighero et al. (1999) showing influences of subconscious priming on grasping reaction times. More recently, evidence that the perception of object orientation is enhanced was shown using eye-movement scanpaths in a visual search task (Bekkering and Neggers 2002). Similar studies have shown comparable effects of action preparation on perception in recent years (Fagioli et al. 2007; Hommel et al. 2001; Wykowska et al. 2009).

Address for reprint requests and other correspondence: T. P. Gutteling, Radboud Univ. Nijmegen, Donders Institute for Brain, Cognition and Behaviour, PO Box 9104, $6500 \mathrm{HE}$ Nijmegen, The Netherlands (e-mail: t.gutteling @ donders.ru.nl).
Specifically, in a recent experiment (Gutteling et al. 2011), we could demonstrate such an effect using a direct measure of visual performance. Subjects performed an orientation change detection task while preparing either a grasping or pointing action toward a bar object. Orientation change perception improved significantly when preparing a grasping action rather than a pointing action. As orientation is a relevant feature for grasping actions, but not for pointing actions, we attribute this visual performance gain to perceptual enhancement of actionrelevant features during motor preparation.

However, much is unclear about the neural implementation of such a mechanism. Recent studies have shown that the frontal eye fields are responsible for shifts of spatial attention known to precede eye movements through connections with early occipital visual areas (V1-V4; Gutteling et al. 2010; Moore et al. 2003; Moore and Fallah 2004; Ruff et al. 2006; Van Ettinger-Veenstra et al. 2009). Here, the modulation of perception is driven by motor preparation in an area that is specialized for a certain action: eye movements. For other actions, such as grasping, this could function similarly: action preparation processes in a specialized cortical visuomotor area might drive perceptual changes in the visual cortex through feedback connections. A recent EEG study (Van Elk et al. 2010) found initial evidence for modulation of occipital activity shortly before grasping, confirming this notion.

A cortical area that has been consistently linked to controlling grasping movements is the anterior part of the intraparietal sulcus (aIPS), which has been well-studied in monkeys (Gallese et al. 1994; Sakata et al. 1995; Taira et al. 1990). One such study (Murata et al. 2000) shows that aIPS consisted of a mix of visual, motor, and visuomotor neurons. Importantly, some neurons showed selectivity for specific object features such as orientation. The human homolog of this area has been established using functional MRI (fMRI; Binkofski et al. 1999; Cavina-Pratesi et al. 2007; Culham et al. 2003), lesion studies (Binkofski et al. 1998), and perturbations using transcranial magnetic stimulation (TMS; Cohen et al. 2009; Dafotakis et al. 2008; Davare et al. 2007; Tunik et al. 2005). Selectivity for relevant object features such as orientation was found in both the visual domain (visual orientation task; Shikata et al. 2001, 2003) and the motor domain (hand/wrist orientation; Taubert et al. 2010; Tunik et al. 2005).

As this area is connected to visual areas (Blankenburg et al. 2010; Nakamura et al. 2001; Ruff et al. 2008), including ventral stream areas (Borra et al. 2008), it is a likely mediator of enhanced orientation sensitivity due to grasping preparation. 
To test the involvement of aIPS in the modulation of orientation perception during action preparation, we aimed to modulate the grasping-induced improvement of orientation perception with TMS on aIPS. That is, we aim to see whether a TMS pulse on aIPS changes this behavioral (perceptual) effect. Therefore, a single pulse of TMS was applied to the left aIPS during grasping or pointing preparation (but before the execution of the action) while subjects performed an orientation change detection task. If aIPS is involved in the modulation of perception during the action preparation phase, stimulation during this phase should have an effect on the enhancement of relevant features induced by action preparation.

\section{MATERIALS AND METHODS}

Participants. Sixteen healthy subjects ( 8 women; mean age $25.6 \mathrm{yr}$, SD 3.4) with normal or corrected-to-normal vision participated in the experiment. All were right-handed as checked by the Edinburgh handedness inventory (mean 81.6, SD 24.1; Oldfield 1971). Subjects signed an informed consent form and went through a TMS safety screening before participation. All procedures were approved by the medical ethical committee of the University Medical Center Utrecht. TMS (resting) motor thresholds were determined per subject by applying TMS to primary motor cortex. Stimulation intensity was decreased whenever a reliable motor response was observed (visually) in any of the fingers for at least 5 out of 10 stimulations (or increased when no such response was found). This was repeated until the motor threshold was reached (Schutter and Van Honk 2006). Mean motor threshold was $56.6 \%$ (SD 8.4) of the maximum machine output.

Apparatus. Subjects sat in a dimly lit room in front of an Iiyama 17-in. $(320-\times 240-\mathrm{mm})$ monitor with a resolution of $1,024 \times 768$ pixels and a refresh rate of $100 \mathrm{~Hz}$. They were seated in a frame with head and chin rest. To minimize head motion during the experiment, a flexible strap was applied around the back of the head. The distance of the frame to the pointing/grasping targets was adjusted per subject to enable comfortable pointing and grasping movements. Visual angles of the stimuli were kept constant by compensating the size of the stimuli relative to the viewing distance.

To ensure that grasping and pointing actions were executed correctly, motion tracking of the right hand (grasping/pointing hand) was performed using the driveBAY magnetic motion tracker (Ascension Technology, Milton, VT). Subjects wore a flexible, unrestrictive glove that was fitted with four motion sensors located at the tip of the thumb, tip of the index finger, back of the hand, and wrist. Movement data were recorded from all sensors at $240 \mathrm{~Hz}$.

TMS was applied using a Magstim Rapid ${ }^{2}$ with a 70-mm figureof-eight coil. During the experiment, subjects received single-pulse (biphasic) stimulation at $110 \%$ of their individual motor threshold.

TMS site localization. MRI-guided navigation was used to place the TMS coil on the appropriate scalp location. T1-weighted anatomic MRI scans were obtained from a Philips 3T Achieva scanner (Philips Medical Systems, Best, The Netherlands) for every participant (scan parameters: echo time/repetition time, $4.6 / 9.87 \mathrm{~ms}$; flip angle, $8^{\circ}$; field of view, $224 \times 160 \times 168 \mathrm{~mm}$; matrix, $256 \times 256$; slice thickness, $1 \mathrm{~mm}$; no slice gap; voxel size, $0.875 \times 0.875 \times 1 \mathrm{~mm}$ ). As aIPS target coordinates (left hemisphere), we used Talairach group activation maximum coordinates [Talairach converted to Montreal Neurological Institute (MNI): $x$ : $-37, y:-45, z: 37]$ reported in an fMRI study by Cavina-Pratesi et al. (2007). In this study, grasping and reaching actions toward three-dimensional objects were contrasted. These coordinates were transformed to native space of individual subjects' brains by inverse normalization using "unified segmentation" (Ashburner and Friston 2005). The TMS targets were manually adjusted by aid of cortical landmarks described in Cavina-Pratesi et al. (2007), where the aIPS was found to be reliably located at the junction of the IPS and the postcentral sulcus; see Fig. 1. The vertex coordi-

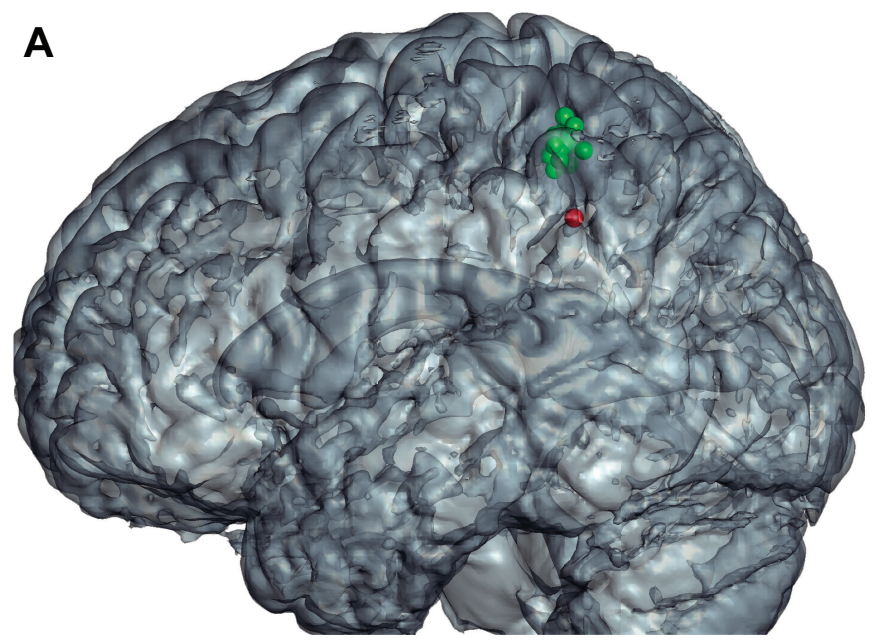

B

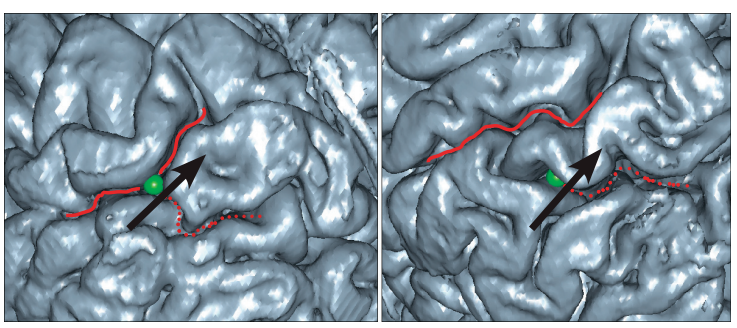

Fig. 1. A: rendering of the renormalized transcranial magnetic stimulation (TMS) targets for all participants on a translucent normalized cortical surface (see MATERIALS AND METHODS) showing individual variability of the TMS targets. The original anterior intraparietal sulcus (aIPS) coordinate from the literature is shown in red. $B$ : renderings of individual TMS target locations, used to guide the TMS coil, and cortical surface for 4 representative subjects. The TMS target was placed at the junction of the aIPS and the postcentral sulcus (PCS). The cortical renderings were directly created from the gray matter segmentation derived from the individual subjects' MRI scan. TMS target locations are shown as green spheres. Dashed lines represent the IPS. Solid lines represent the PCS. Arrows indicate the estimated optimal TMS current direction to which the TMS coil was aligned during placement.

nates were determined purely based on anatomic landmarks and defined as the top-most part of the head, between hemispheres.

Before the experiment, the anatomic scan was registered with the subject's actual head, using a stereotactic neural navigation system (NeNa 2.0; Brain Science Tools, Utrecht, The Netherlands) in combination with the driveBAY magnetic tracker, for the measurement of facial landmarks. Coil placement markings were drawn on a tightly fitting swimming cap covering the subject's head. In addition, the orientation of the aIPS was marked. The coil was placed such that it was tangential to the scalp and the current induced in the brain is perpendicular to the sulcal direction to optimize stimulation efficiency; see Fig. $1 B$. It is thought that the direction of induced current and the general direction of the underlying pyramidal neuron tracts are roughly the same in this situation, yielding optimal TMS effects (Kammer et al. 2007; Mills et al. 1992). In the vertex (control) condition, the coil was placed on the top of the head, with the focal point of stimulation in between hemispheres and the induced current directed along the hemispheric division. As stimulation of this area is very ineffective, no neural effect is expected from this stimulation condition.

For statistical purposes, the native space target coordinates were (re)normalized to MNI space. All TMS targets fitted within a sphere with radius $6.3 \mathrm{~mm}$ (maximum distance to mean coordinates). The mean stimulation coordinates (MNI: $x$ : $-42.5, y:-42.8, z: 51.9)$ were located $16.1 \mathrm{~mm}$ (Euclidean distance) from the original coordinate (MNI: $x$ : -37 , 
$y:-45, z: 37)$ from the literature, mainly due to the elevation toward the surface of the brain.

Task. Subjects were instructed to perform an orientation change detection task (see Fig. 2) while performing grasping or pointing actions with the right (dominant) hand, similar to the task described in Gutteling et al. (2011). Every trial started with a blue fixation spot (2,000-2,500 $\mathrm{ms}, 0.7^{\circ}$ diameter of visual angle), after which a red rectangular bar appeared $\left(0.8 \times 4^{\circ}\right.$ visual angle $)$ for $130 \mathrm{~ms}$ in any of four locations, equidistant $\left(9^{\circ}\right.$ from fixation to bar center) to the fixation spot. After a brief disappearance $(100 \mathrm{~ms})$, the bar reappeared in the same location, either slightly rotated or having the same angle. At this point, the fixation spot disappeared, and fixation was no longer required. We verified previously (pilot study, Gutteling et al. 2011), using eye tracking, that subjects could fixate while performing this task. Without time pressure, subjects indicated by pressing one of two keys with their left hand whether they had observed a difference in orientation between the first and second presentation of the bar. The bar stayed on screen until a response was given. Simultaneously, subjects were required to perform either a grasp or point action with their right hand to the appearing bar, depending on the instruction at the start of the block. Thus the grasping or pointing action was performed using the right hand while the response to the discrimination task was given using the left hand. The go-cue for this action was the first appearance of the bar. Subjects were specifically instructed to initiate the action as soon as the first bar appeared. This realizes a situation where the to-be discriminated orientation change occurs during the grasping preparation phase, as the orientation change occurs well before the grasping/pointing movement onset (pointing/ grasping movements have latencies of $\sim 400 \mathrm{~ms}$; see Neggers and Bekkering 1999; Prablanc et al. 1986). Grasping was performed by applying a "precision grip" in the length direction of the bar, i.e., to place index finger and thumb at the opposing short sides of the bar. As subjects are not grasping a real-life object (it is merely drawn on the screen), the precision grip cannot be fully applied, but we verified previously (Gutteling et al. 2011) that the kinematics in this situation are comparable with a real precision grip. The pointing action involved pointing to the center of the bar with the index finger. As both actions contain a transport component, these are essentially reach-tograsp and reach-to-point actions. When a successful (determined by comparing previously recorded target coordinates) grasping or pointing action was performed, the bar turned green and a sound was played. This was done to motivate participants to make correct
A

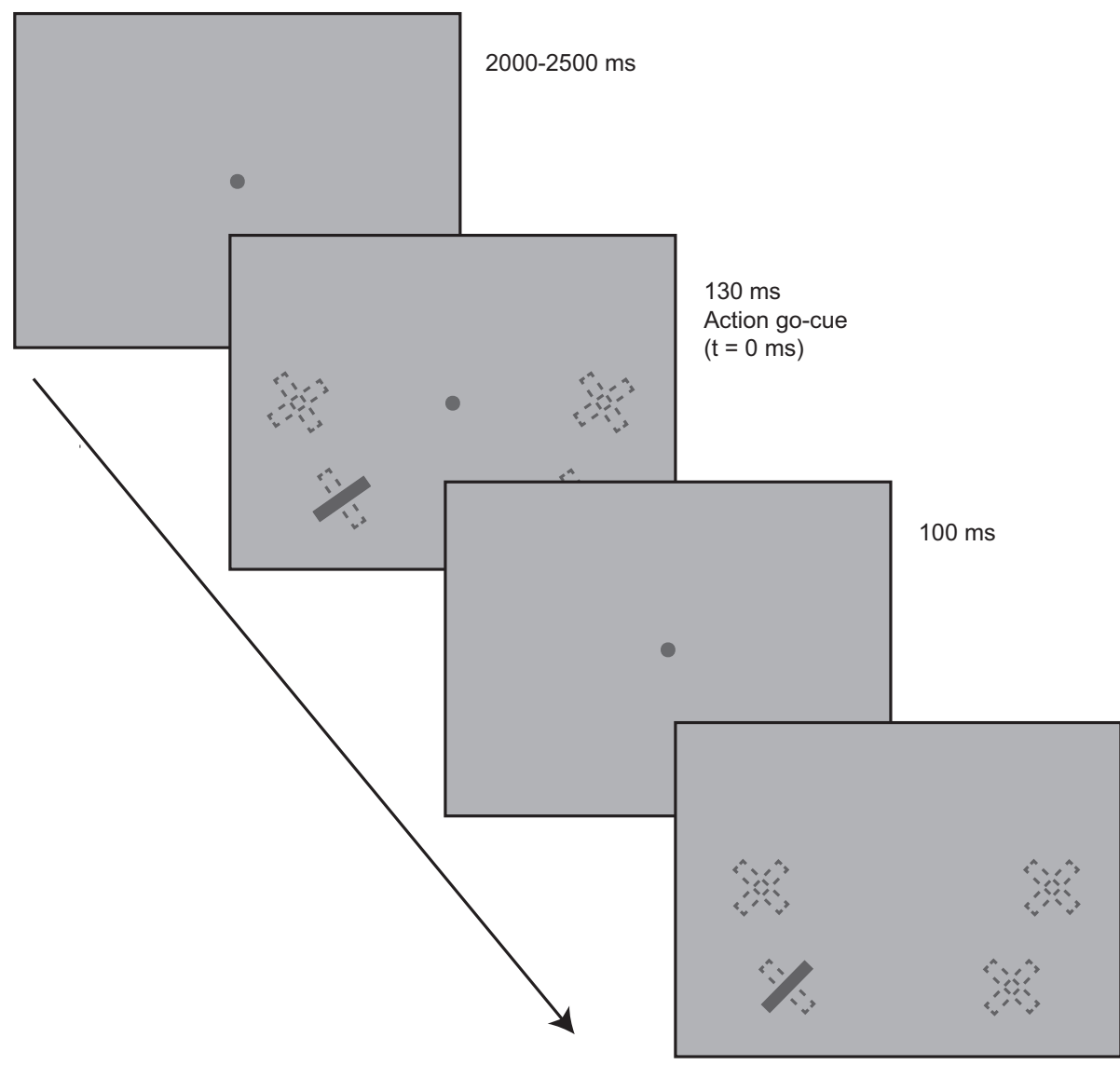

Fig. 2. A: schematic representation of the orientation change detection task. Subjects were presented with a blank screen for 2,000-2,500 $\mathrm{ms}$ (random length) after which the $1 \mathrm{st}$ bar appeared at any of 4 locations, equidistant to the fixation spot (indicated by the dashed outlines), which served as the grasping or pointing go-cue. This bar could have an orientation differing from $\pm 45^{\circ}$ (a large $5^{\circ}$, small $3^{\circ}$, or no difference, and hence this 1 st bar could be oriented at $\pm 40,50,42,48$, or $45^{\circ}$ ) and disappeared after $130 \mathrm{~ms}$. After a blank screen for $100 \mathrm{~ms}$, another bar appeared and stayed on screen that was always $\pm 45^{\circ}$. Because of grasping/pointing latencies, the instructed action was not executed until after the appearance of the 2nd bar. After execution of the action, subjects could respond by key press whether they had perceived a change without time pressure. $B$ : timeline of events. $T=0$ represents the action go-cue. Single-pulse TMS was delivered at $t=-40,0$, or +40 relative to the go-cue ( $1 \mathrm{st}$ bar appearance).

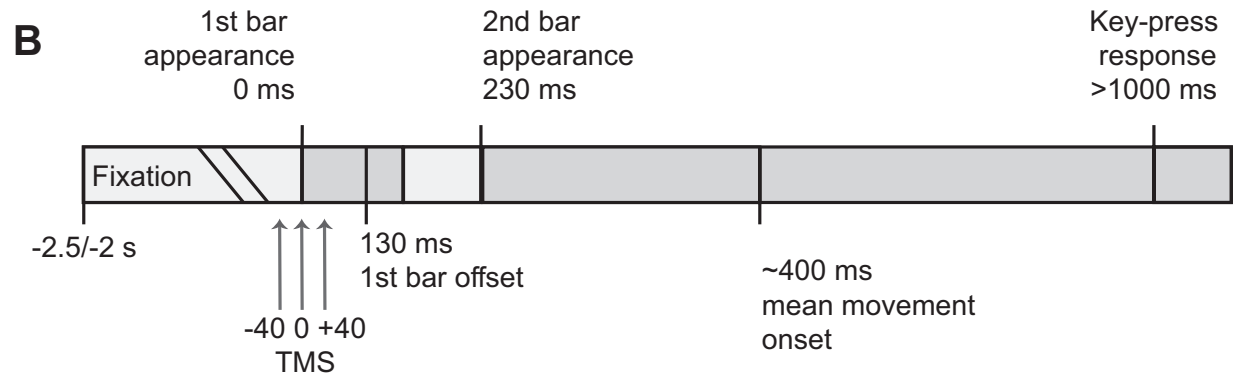

J Neurophysiol • doi:10.1152/jn.00622.2012 • www.jn.org 
grasping or pointing movements. Online analysis of the grasping or pointing movement data (acquired by the motion tracker) was performed to determine whether the action was executed correctly.

The difference in orientation between the 1 st and 2 nd bar could be "none," "small" ( $3^{\circ}$ rotation), or "large" $\left(5^{\circ}\right)$. The 2 nd bar was always oriented at either 45 or $-45^{\circ}$ (and hence the 1 st bar at $\pm 40,50,42$, 48 , or $45^{\circ}$ ). In half of the trials, an orientation difference occurred, either small or large (also distributed equally). These differences occurred in both a clockwise and counterclockwise direction. Movement onset time (when the wrist speed surpassed $0.15 \mathrm{~m} / \mathrm{s}$ ) was monitored to check whether no movement was made before the second bar appeared to ensure that the discrimination was made in the action preparation phase. In case this was violated, the trial was discarded during the analysis. This data rejection, based on movement onset times, yielded no significant differences in trial rejection rates between sites (aIPS, vertex) or actions (grasping, pointing). The mean trial rejection rate in the aIPS stimulation condition was $2.93 \%$ (SD 1.41) for grasping and $2.24 \%$ (SD 1.27) for pointing. In the vertex condition, the mean rejection rate was $2.44 \%$ (SD 0.94) for grasping and $2.39 \%$ (SD 1.58) for pointing.

In every trial, a single pulse of TMS was applied to the left aIPS or the vertex at any one of three different time points around the onset of the first bar: $40 \mathrm{~ms}$ before $(-40)$, during $(0 \mathrm{~ms})$, or $40 \mathrm{~ms}$ after $(+40)$ the first bar appearance. The first rather than the second bar was chosen because the first bar contains the information necessary to make the orientation change detection, i.e., only the first bar could have an orientation deviating from $\pm 45^{\circ}$. The second bar is always oriented at $\pm 45^{\circ}$ to ensure that the grasping itself was always to a bar oriented at either -45 or $45^{\circ}$.

The stimulation times were chosen based on estimated cortical conduction times for visual input (the time between presentation of the 1st bar stimulus and its arrival in the visual cortex) and between aIPS and the visual areas. We estimated these times to be roughly equal (see Neggers et al. 2007 for a similar reasoning), i.e., assuming feedback connections between aIPS and the visual cortex, a TMS pulse and a visual stimulus at time 0 should arrive simultaneously in the visual cortex. Given the hypothesis that a feedback signal arises from aIPS during early action preparation that subsequently modulates perception in the visual cortex before or during visual input, we centered the timing of the TMS pulses on $0 \mathrm{~ms}$ (onset of the 1st bar stimulus).

Before starting the actual experiment, subjects were trained to reach adequate orientation change detection performance levels. The previous study using the same task (Gutteling et al. 2011) showed that subjects needed a certain performance level to show the effect of action preparation on perception in the results. We aimed to get subjects to this adequate level of performance before starting the TMS experiment. On average, subjects completed 4-5 training blocks ( 32 trials each) before starting the actual experiment. Subjects were instructed to respond conservatively, i.e., to prevent false alarm at the cost of hits. Detection performance was checked after each training block. Criteria for "adequate performance" were a hit rate (HR) in the small change condition of $25 \%$ or better, better performance on the "large change" condition (large change $>$ small change), and a false alarm rate (FA) of $<40 \%$. After training, subjects completed 8 blocks of 64 trials each. Site of stimulation (aIPS, vertex) was changed after 4 blocks and was counterbalanced across subjects. Grasping and pointing blocks alternated, and the order was also counterbalanced across subjects.

Stimuli were presented using custom software (Trackmagic, written in $\mathrm{C}++$ ) that was also able to interface both with the movement tracker for synchronized data acquisition and the TMS device for triggering the TMS pulse. Care was taken to ensure accurate timing of stimulus presentation and pulse triggering by synchronizing to the screen refresh rate of the display monitor.
Behavioral analysis. All analyses were done using custom MATLAB scripts (The MathWorks, Natick, MA). Statistical analyses were performed using SPSS 15.0 (Chicago, IL).

Sensitivity $\left(\mathrm{d}^{\prime}\right)$ for each TMS site/TMS timing/action combination was estimated by subtracting $z$-transformed HR and FA $\left[\mathrm{d}^{\prime}=\right.$ $Z(\mathrm{HR})-Z(\mathrm{FA})$, where $Z()$ is the $z$-transformation, which is the inverse cumulative normal distribution under the equal variance assumption]. Sensitivity values were calculated individually per subject and separately for every block. Values for the same conditions were averaged over blocks afterward. This was done to prevent any difference in response bias between blocks from contaminating the performance measure.

These $d^{\prime}$ values were analyzed in a repeated-measures ANOVA with the factors TMS SITE (aIPS, vertex), TMS TIMING $(-40,0$, +40 ), and ACTION (grasping/pointing) for all effects of interest. Additionally, post hoc tests were performed, which were mainly illustrative as to the nature of the interactions found in the main analysis. We performed these post hoc tests separately and independently only when there was a significant main effect or interaction. For these tests, no multiple-comparisons correction was applied. However, this has no implications for the significance of the main findings, as they were based on an ANOVA that implicitly corrects for multiple comparisons.

Kinematics. To test whether stimulation of aIPS had direct effects on the grasping and pointing movements, motion tracking of the grasping/pointing hand was performed during the performance of the task. Kinematic data were obtained using a magnetic motion tracker (driveBAY).

Parameters were extracted from the acquired movement data, including movement onset, duration, (time to) peak velocity, grasp angle, and aperture. Grasp angle was calculated as a two-dimensional projected angle between the index finger-thumb pair and an imaginary vertical line on the monitor. Grasping aperture was the distance between these fingers. The grasping angle time course (from movement onset to offset) was divided into 24 time bins and tested for significant differences between aIPS and vertex stimulation conditions per timing and target orientation.

Remaining parameters were extracted from the wrist probe. Movement onset threshold was set at $0.15 \mathrm{~m} / \mathrm{s}$.

All parameters were entered in an ANOVA with factors TMS SITE, TIMING, and ACTION.

Reaction times to the key-press response were not analyzed, as this response was nonspeeded.

Visual hemifield. In the preceding study (Gutteling et al. 2011), we found an effect of visual hemifield, in that the enhancement in orientation detection due to grasping preparation was only found for the right visual field. To test whether this effect is present in the current data and whether it is affected by TMS, the current data were also divided by hemifield in which the stimulus appeared. As fixation was required until the second bar appeared, stimuli presented on the left side of the screen appeared in the subject's left visual field (and vice versa). The data were divided by visual hemifield (left/right), site of stimulation (aIPS/vertex), and action (grasping/pointing) but collapsed over TMS timing $(-40 / 0 /+40)$ to retain sufficient trials to estimate a reliable sensitivity measure. These $\mathrm{d}^{\prime}$ values were used in a repeated-measures ANOVA with the factors HEMIFIELD, TMS SITE, and ACTION.

Control experiment. Although we assume that the orientation change discrimination made in the main task is based on the comparison of the 2 bar presentations (the 2 nd being the reference), it may be possible to perform the task with only the 1 st bar presentation. To check for this possibility, a control experiment was performed.

Six participants participated in this control experiment. Subjects were first trained on the original task, to achieve adequate performance level, according to the requirements of the main task (see methods above). Subjects then performed 128 trials of the original task without performing a grasping or pointing action (only a key 


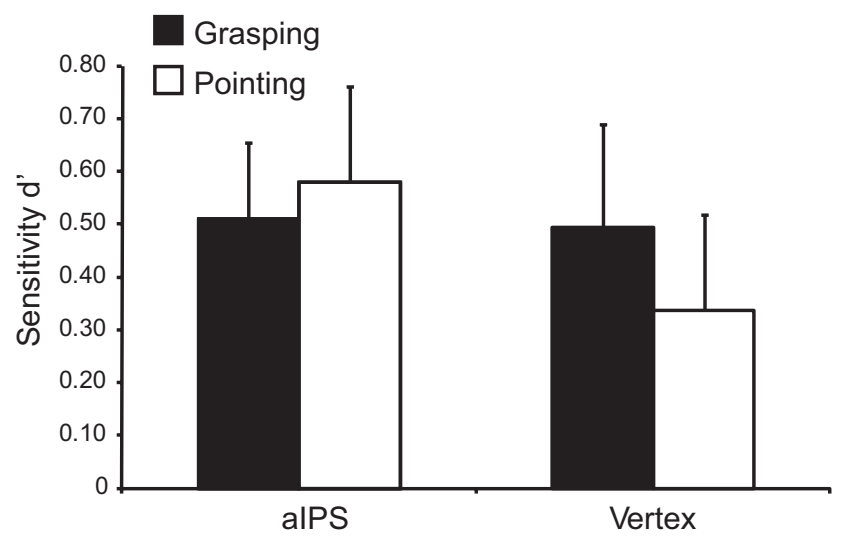

Fig. 3. Mean sensitivity values $\left(\mathrm{d}^{\prime}\right)$ for the aIPS and Vertex stimulation conditions during grasping and pointing preparation. Error bars represent the standard error.

press). After this, subjects performed another 128 trials but without the 2 nd bar presentation. Sensitivity values were estimated for large and small changes for both single and dual bar conditions.

\section{RESULTS}

Subjects. Two of the sixteen participants were excluded due to poor (perceptual) task performance. Of the remaining fourteen participants, two showed a strong opposite behavioral effect to what was found in an earlier study using the same paradigm (Gutteling et al. 2011) in the control condition (vertex TMS) averaged over all timings (although a net effect over all subjects was still present). That is, they showed enhanced orientation perception when preparing a pointing action. The current study aims to use TMS to modulate the specific effect of grasping-induced orientation discrimination enhancement, and thus these two subjects were eliminated. This did not alter the direction of the effects found in the initial analyses but did increase specificity of the effects.

Main analysis. A significant SITE $\times$ ACTION interaction was found $\left[F_{(1,11)}=6.97, P=0.023\right.$, partial $\left.\eta=0.39\right]$, see Fig. 3 . This indicates that, as hypothesized, the effect of action preparation significantly differs between stimulation sites. Mean discrimination performance values in the control condition (vertex), pooled over timing, are higher when preparing a grasping action than a pointing action (grasping $\mathrm{d}^{\prime}=0.49, \mathrm{HR}=42.9 \%, \mathrm{FA}=$ $25.5 \%$, pointing $\mathrm{d}^{\prime}=0.34, \mathrm{HR}=39.7 \%, \mathrm{FA}=27.5 \%$ ). However, with aIPS stimulation, performance in the grasping condition is not higher than in the pointing condition (grasping $\mathrm{d}^{\prime}=0.51$, $\mathrm{HR}=39.8 \%, \mathrm{FA}=21.7 \%$; pointing $\mathrm{d}^{\prime}=0.58, \mathrm{HR}=45.9 \%$, $\mathrm{FA}=24.6 \%$ ), effectively negating the action-induced perceptual modulation observed in our previous study and in the control TMS condition.

Although mean sensitivity in the aIPS stimulation condition seemed higher than in the control condition (aIPS: 0.54, SD 0.19 ; vertex: 0.41 , SD 0.22), this effect was not significant as no significant main effect of SITE was found $\left[F_{(1,11)}=1.56\right.$, $P=0.24$, partial $\eta=0.12]$. In a direct comparison per action, paired-samples $t$-tests show that, although not significant, the main difference between sites is between pointing-aIPS stimulation and pointing-control (paired-samples $t$-test grasping aIPS vs. control: $t=0.17, P=0.87$; pointing aIPS vs. control: $t=1.9, P=0.08$ ).
In addition, a significant main effect of TIMING was found $\left[F_{(2,22)}=5.00, P=0.019\right.$, partial $\left.\eta=0.31\right]$, indicating that there was a significant difference in visual perception performance depending on the time of stimulation. Post hoc pairwise comparisons showed that performance in the "stimulus onset" $\left(0 \mathrm{~ms} ; \mathrm{d}^{\prime}=0.66\right.$, SD 0.65$)$ stimulation condition was significantly increased relative to early $\left(-40 ; \mathrm{d}^{\prime}=0.36\right.$, SD 0.55$)$ stimulation $(P=0.021)$ and late $\left(+40 ; \mathrm{d}^{\prime}=0.42\right.$, SD 0.61$)$ stimulation $(P=0.039)$. This may simply be due to the cooccurrence of the TMS pulse and the stimulus presentation, as there was no significant interaction with stimulation site $\left[F_{(2,22)}=1.64, P=0.54\right.$, partial $\left.\eta=0.05\right]$ and it is therefore unlikely to be a neural effect of interest. It may be that this reflects an alerting effect or even the addition of (sensory) noise (Lugo et al. 2008; Moss et al. 2004).

Effects of visual hemifield. In a separate analysis, the data were pooled over timings and separated by hemifield where the stimulus appeared to investigate the effect of visual field on discrimination performance. See Fig. 4 for an overview of the results. The data were collapsed over timing to retain sufficient trials, as no interaction with TIMING was found in the main analysis. This ANOVA yielded a significant SITE $\times$ ACTION interaction $\left[F_{(1,11)}=5.83, P=0.034\right.$, partial $\left.\eta=0.35\right]$, as was found in the main analysis (see above). In addition, a main effect of hemifield was found at trend level $\left[F_{(1,11)}=3.82\right.$, $P=0.076$, partial $\eta=0.26$, where sensitivity was generally higher for stimuli in the left visual field (LEFT: $\mathrm{d}^{\prime}=0.65$, RIGHT: $\left.d^{\prime}=0.48\right)$. In one-tailed paired-sample $t$-tests per hemifield, a significant difference $(t=2.02, P=0.034)$ between grasping and pointing performance was only found in the vertex-right condition (in favor of grasping), indicating that the effect of action preparation may only occur in the right hemifield.

Hand movement kinematics. Analysis of the extracted movement parameters, using an ANOVA with the same factors as the main analysis above, yielded a significant main effect of TIMING for movement onset $\left[F_{(2,10)}=4.80, P=0.019\right.$, partial $\eta=0.30]$. Stimulation in the +40 condition resulted in a delayed movement initiation ( $610 \mathrm{~ms}$ for +40 compared with $587 \mathrm{~ms}$ for -40 and $585 \mathrm{~ms}$ for $0 \mathrm{~ms}$ ). As there is no effect of stimulation site $\left[F_{(1,11)}=2.90, P=0.117\right.$, partial $\left.\eta=0.21\right]$, this likely reflects a secondary nonneuronal TMS effect such as

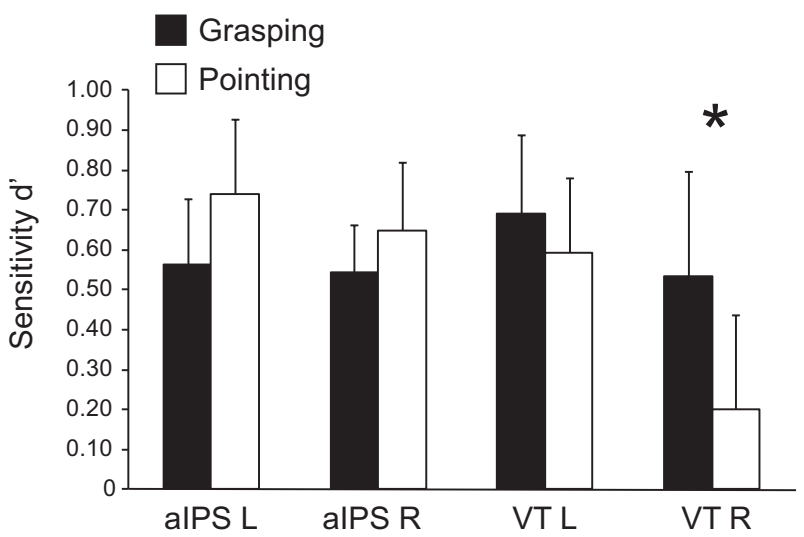

Fig. 4. Mean sensitivity values (ordinate) separated by site of stimulation and visual field that the stimulus was presented in. VT, vertex; L, left visual field; $\mathrm{R}$, right visual field. $* P<0.05$, paired-sample $t$-test. Error bars represent the standard error. 
a general increase in movement initiation time simply due to the warning signal provided by the late TMS pulse relative to the go-cue. Movement onset times did not differ significantly between grasping and pointing actions $\left[F_{(1,11)}=0.12, P=\right.$ 0.735 , partial $\eta=0.11]$, suggesting a similar motor preparation time course.

Peak velocity values significantly differed between grasping and pointing actions [main effect of ACTION: $F_{(1,11)}=17.34$, $P=0.002$, partial $\eta=0.61]$, where grasping $(71.2 \mathrm{~cm} / \mathrm{s}, \mathrm{SD}$ 16.9) was faster than pointing (63.6 cm/s, SD 17.7).

A significant TIMING $\times$ SITE interaction was found for the "time-to-peak velocity" parameter $\left[F_{(2,22)}=5.63, P=0.011\right.$, partial $\eta=0.34]$. This, however, did not differ between actions $\left[\right.$ SITE $\times$ ACTION $\times$ TIMING: $F_{(2,22)}=0.254, P=0.778$, partial $\eta=0.23$ ] and is therefore not a factor in grasping/ pointing performance differences.

No significant factors were found in the "maximum grasping aperture" (all $F<0.35, P>0.57$ ) or "movement duration" (all $F<3.39, P>0.093$ ) ANOVAs. Analysis of grasping angle time courses did not reveal any significant differences between aIPS and control stimulation for any timing or target angle, i.e., no effect of TMS was found on the angle preshaping during the grasping action.

In summary, for the timings tested, no significant effects of aIPS stimulation were observed on grasping or pointing kinematics specifically.

Control experiment. A control experiment was performed to check whether the task could be performed with only a single bar presentation. See Fig. 5 for an overview of the results. Interestingly, and contrary to our expectations, subjects were able to discern orientations deviating from 45 or $-45^{\circ}$ based on only the first bar presentation, albeit with lower performance. The $\mathrm{d}^{\prime}$ for large orientation changes $\left(5^{\circ}\right)$ was 1.22 (SD 0.49 ) with and 1.06 (SD 0.37) without the second bar. For the small orientation changes $\left(3^{\circ}\right), \mathrm{d}^{\prime}$ was 0.61 (SD 0.47) with the 2nd bar and 0.45 (SD 0.40) without. All d' values were significantly above 0 (1-sample $t$-test vs. 0$)$. Thus subjects were able to detect (absolute) bar orientations deviating from $45^{\circ}$.

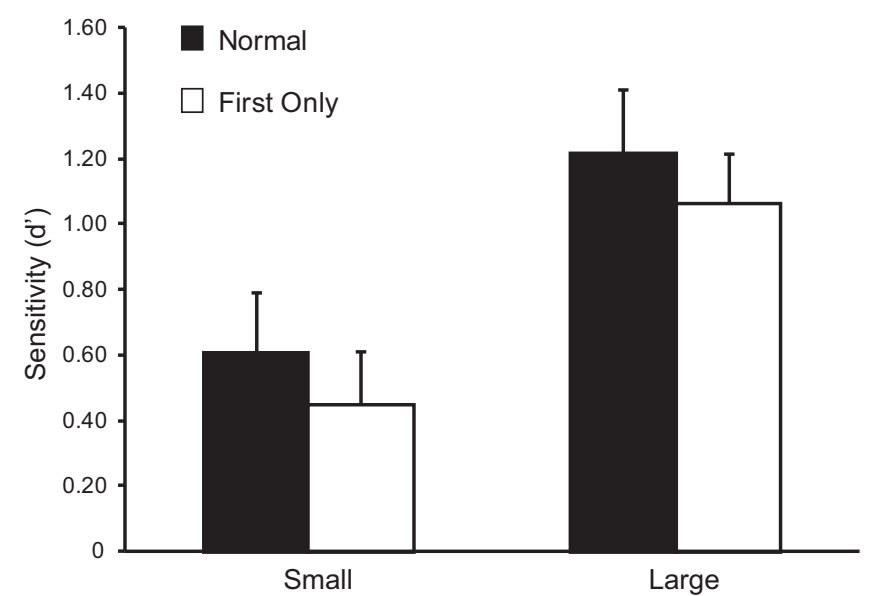

Fig. 5. Results from the control experiment. In the normal condition, the task was identical to that used in the main experiment, with 2 bar presentations. In the First Only, no secondary bar was presented, and the orientation change detection had to be made based on the 1st bar presentation only. Small and Large refer to the magnitude of orientation deviation from $\pm 45^{\circ}$ (Small: $3^{\circ}$; Large: $5^{\circ}$ ). Error bars represent the standard error.

\section{DISCUSSION}

In the current study, we aimed to investigate the neural mechanism underlying action-modulated perception phenomena that have been reported for arm and hand movements in several studies (Bekkering and Neggers 2002; Craighero et al. 1999; Gutteling et al. 2011; Wykowska et al. 2009). We hypothesized that the aIPS is involved in not only the preparation and control of grasping actions, but also the modulation of perception of visual object features that are relevant for the upcoming action. We found that stimulation of the left aIPS selectively modulates orientation sensitivity during action preparation compared with control stimulation (vertex). This argues for the involvement of aIPS as a critical area in the mechanism underlying the effect of action-modulated perception and that aIPS might influence perception through cortical feedback connections to the visual areas.

Stimulation of aIPS early during the movement preparation phase affected object perception, but no effects were found on the execution of grasping or pointing itself. Effects of aIPS TMS on grasping kinematics have been shown previously for TMS applied both during the movement (Cohen et al. 2009; Dafotakis et al. 2008; Tunik et al. 2005) and at the grasping go-cue (Davare et al. 2007). In the latter study by Davare and colleagues (2007), effects on hand preshaping were found with bilateral aIPS stimulation at $140 \%$ of the resting motor threshold. However, no effects on kinematics have been reported using unilateral, single-pulse stimulation at the go-cue, as is the case in the current study. It is likely that we stimulated aIPS too early in the grasping preparation period or too weak to affect the kinematics significantly. This may have resulted in effects being absent or too weak to be detected with the current sample size. Furthermore, it is possible that kinematic effects were present, such as modulation in grip force scaling or endpoint dispersion, but not measured in the current setup [cathode ray tube (CRT) monitor interference precluded exact measurements of kinematic endpoints]. Also, we do believe aIPS plays an important role in the hand shaping while grasping despite the two-dimensional objects to be grasped. Orientation is a relevant parameter for the grasping but not the pointing action in our study. Although we do not believe that the lack of kinematic effects is due to mislocalization of aIPS, it may be beneficial for the effectivity of TMS to localize aIPS functionally by testing for kinematic effects or by localizing individual aIPS functionally using fMRI.

In the previous study (Gutteling et al. 2011), an effect of visual hemifield was found where the selective enhancement due to grasping preparation was only present when stimuli were presented in the right visual field, possibly because subjects grasped with their right hand. In the current study, this effect was not significant but appeared as a trend. In a direct comparison between actions, a significant difference was found in the vertex condition, for the right visual hemifield. In the aIPS stimulation condition, no significant effect of motor preparation on detection performance was found for either left or right visual field. Obviously, as no effect of motor preparation was found in the left visual field, there was no modulation of this effect with TMS. The effect of aIPS TMS reported in the current study may predominantly be based on effects in the right visual field. This might not be surprising, as the left aIPS was stimulated and all movements were made with the right 
hand. Based also on the hemifield effect in the preceding study, we believe that neuronal signals from aIPS, contralateral to the moving hand, are relayed to the visual cortex in the same hemisphere to manipulate visual processing during action preparation. Influences of TMS on action-modulated perception can then be expected in the hemifield ipsilateral to the moving hand and contralateral to the stimulated hemisphere. Note that the movements were made with the dominant hand, which was the right hand for all participants. It is unclear whether this effect depends on the hand used for action execution or hand dominance.

In a separate control experiment, we found that subjects were actually able to maintain an above-chance level of performance when performing the task with only a single bar presentation. Although this is an unexpected finding, we do not believe this poses problems for the interpretation of the results in our study. The timing of the TMS pulses was based around the first bar presentation, which we believed to be the moment of orientation discrimination (i.e., the moment when high orientation sensitivity was most essential). The results from this control experiment do not disprove this. In fact, they confirm this notion. It may be that participants used an internal reference to the target $\left(45^{\circ}\right)$ orientation. This does not affect the notion that subjects performed an orientation judgment on the first bar stimulus (which is enhanced when grasping compared with pointing). In general, we do not believe this (unexpected) finding in the control experiment poses a problem for the proposed mechanism of action-modulated orientation perception. It might just be that to some extent the perception of an absolute orientation was modulated by the action rather than perception of an orientation change.

It is unlikely that the differences in detection performance found in the current study are due to differences in the difficulty of grasping or pointing actions. No differences in orientation sensitivity between grasping and pointing conditions occur when an action-irrelevant feature (luminance) is used as the to-be discriminated feature (Gutteling et al. 2011).

The observed modulation of orientation discrimination sensitivity when stimulating aIPS is compatible with the idea of neuronal feedback connections from aIPS to the visual cortex modulating perception, as put forward in the introduction. This can be regarded as a top-down attentional effect, enhancing relevant features (orientation) over others. There are many different forms of top-down attentional sources; we propose that action preparation is such a specific source.

It is interesting to note that, although not reaching significance level, mean overall discrimination sensitivity, irrespective of the action being prepared, was also found to be higher when stimulating aIPS compared with vertex stimulation as can be seen in Fig. 3. When comparing the sensitivity values between sites in this figure and the post hoc paired-sample $t$-test, one can observe that pointing sensitivities seem to differ between sites, whereas grasping sensitivities do not. As we aimed to affect the enhancement of orientation sensitivity due to grasping preparation, this finding may seem counterintuitive. However, these observed effects can be explained in two ways.

First, aIPS stimulation may cause a general increase in performance (i.e., elevated baseline performance for both grasping and pointing) by connections from the IPS to the visual cortex combined with a disruption of the signal that causes grasping-induced perceptual enhancement through an- other feedback channel. As functional areas have numerous connections to various brain areas, it is plausible to assume that TMS (which has been shown to activate remotely connected areas) stimulates more than one functional channel. In our own laboratory, we have found general improvements of discrimination performance due to single-pulse stimulation of the IPS before (Van Ettinger-Veenstra et al. 2009), which is consistent with existing literature (Blankenburg et al. 2010; Ruff et al. 2008). Similarly, general perceptual discrimination improvements have been found for TMS (Grosbras and Paus 2003; Ruff et al. 2006; Van Ettinger-Veenstra et al. 2009) and microstimulation (Moore and Fallah 2004) of the frontal eye fields as well. It is at present not clear what the general effect of a single pulse of TMS is in the brain. This seems to depend on many factors, such as pulse parameters, coil orientation, and the neuronal state at the moment of stimulation (Sandrini et al. 2011).

Second, TMS on aIPS may have a single effect by enhancing perceptual sensitivity only in the pointing condition. In this case, TMS on the aIPS may add a feedback signal during pointing preparation to the visual cortex, similar to the aIPS feedback signal naturally occurring during grasping preparation. Thus the stimulation of aIPS during pointing preparation may mimic the feedback signals normally sent to the visual cortex during grasping preparation. This can result in similar discrimination performance increases during pointing preparation as is usually observed during grasping preparation. Stimulation of aIPS during grasping preparation might not further enhance orientation sensitivity (e.g., a ceiling effect). Consequently, this diminishes the performance differences between pointing and grasping in the aIPS stimulation condition.

Both these possible explanations fit with the idea of cortical feedback connections between motor area aIPS and visual areas that are activated when preparing an action, as hypothesized. Recently, an EEG study (Van Elk et al. 2010) demonstrated enhanced activation in the visual cortex for grasping compared with pointing, which can be regarded as first evidence for influences of motor areas controlling grasping on processing in the visual cortex. This mechanism may be similar to the cortical feedback connections between the frontal eye fields and occipital areas, enabling an enhancement of spatial perception during the preparation of an eye movement (Gutteling et al. 2010; Ruff et al. 2006; Van Ettinger-Veenstra et al. 2009). Both types of action preparation (for eye movements and grasping movements) may induce some sort of actionmodulated perception where the preparation of a certain action also entails the perceptual enhancement of features that are relevant for the upcoming action, increasing its chance of success.

The current study shows that the anterior intraparietal area is involved in the perceptual modulation preceding a manual action, just as the frontal eye fields are involved in the modulation of perception preceding eye movements. It is unlikely that aIPS is the sole contributor to the perceptual effects during action preparation. For task used in the current study, where orientation is a relevant feature, one may consider the involvement of other brain areas somehow involved in aspects of orientation coding for motor planning. Of particular interest is the monkey area V6(A) (Fattori et al. 2010; Galletti et al. 2003) and its likely human homolog, the superior parietooccipital cortex (SPOC; Gallivan et al. 2009; Monaco et al. 2011), both 
of which have been shown to be important for wrist orientation while grasping. Further studies are necessary not only to show the exact mechanism that drives the perceptual changes due to action preparation, but also to identify these other motor areas involved in the general mechanism of action-modulated perception.

\section{GRANTS}

This work was supported by a Netherlands Organisation for Scientific Research (NWO) Open Competition Grant NWO 400-05-134.

\section{DISCLOSURES}

No conflicts of interest, financial or otherwise, are declared by the author(s).

\section{AUTHOR CONTRIBUTIONS}

T.P.G., J.L.K., and S.F.W.N. conception and design of research; T.P.G. and S.Y.P. performed experiments; T.P.G. and S.Y.P. analyzed data; T.P.G., S.Y.P., J.L.K., and S.F.W.N. interpreted results of experiments; T.P.G. prepared figures; T.P.G. drafted manuscript; T.P.G., J.L.K., and S.F.W.N. edited and revised manuscript; T.P.G., S.Y.P., J.L.K., and S.F.W.N. approved final version of manuscript.

\section{REFERENCES}

Ashburner J, Friston KJ. Unified segmentation. Neuroimage 26: 839-851, 2005.

Bekkering H, Neggers SF. Visual search is modulated by action intentions. Psychol Sci 13: 370-374, 2002.

Binkofski F, Buccino G, Stephan KM, Rizzolatti G, Seitz RJ, Freund HJ. A parieto-premotor network for object manipulation: evidence from neuroimaging. Exp Brain Res 128: 210-213, 1999.

Binkofski F, Dohle C, Posse S, Stephan KM, Hefter H, Seitz RJ, Freund HJ. Human anterior intraparietal area subserves prehension: a combined lesion and functional MRI activation study. Neurology 50: 1253-1259, 1998.

Blankenburg F, Ruff CC, Bestmann S, Bjoertomt O, Josephs O, Deichmann R, Driver J. Studying the role of human parietal cortex in visuospatial attention with concurrent TMS-fMRI. Cereb Cortex 20: 2702-2711, 2010.

Borra E, Belmalih A, Calzavara R, Gerbella M, Murata A, Rozzi S, Luppino G. Cortical connections of the macaque anterior intraparietal (AIP) area. Cereb Cortex 18: 1094-1111, 2008.

Cavina-Pratesi C, Goodale MA, Culham JC. FMRI reveals a dissociation between grasping and perceiving the size of real 3D objects. PLoS One 2: e424, 2007.

Cohen NR, Cross ES, Tunik E, Grafton ST, Culham JC. Ventral and dorsal stream contributions to the online control of immediate and delayed grasping: a TMS approach. Neuropsychologia 47: 1553-1562, 2009.

Craighero L, Fadiga L, Rizzolatti G, Umilta C. Action for perception: a motor-visual attentional effect. J Exp Psychol Hum Percept Perform 25: 1673-1692, 1999.

Culham JC, Danckert SL, DeSouza JF, Gati JS, Menon RS, Goodale MA. Visually guided grasping produces fMRI activation in dorsal but not ventral stream brain areas. Exp Brain Res 153: 180-189, 2003.

Dafotakis M, Sparing R, Eickhoff SB, Fink GR, Nowak DA. On the role of the ventral premotor cortex and anterior intraparietal area for predictive and reactive scaling of grip force. Brain Res 1228: 73-80, 2008.

Davare M, Andres M, Clerget E, Thonnard JL, Olivier E. Temporal dissociation between hand shaping and grip force scaling in the anterior intraparietal area. J Neurosci 27: 3974-3980, 2007.

Fagioli S, Hommel B, Schubotz RI. Intentional control of attention: action planning primes action-related stimulus dimensions. Psychol Res 71: 22-29, 2007.

Fattori P, Raos V, Breveglieri R, Bosco A, Marzocchi N, Galletti C. The dorsomedial pathway is not just for reaching: grasping neurons in the medial parieto-occipital cortex of the macaque monkey. J Neurosci 30: 342-349, 2010.
Gallese V, Murata A, Kaseda M, Niki N, Sakata H. Deficit of hand preshaping after muscimol injection in monkey parietal cortex. Neuroreport 5: 1525-1529, 1994.

Galletti C, Kutz DF, Gamberini M, Breveglieri R, Fattori P. Role of the medial parieto-occipital cortex in the control of reaching and grasping movements. Exp Brain Res 153: 158-170, 2003.

Gallivan JP, Cavina-Pratesi C, Culham JC. Is that within reach? fMRI reveals that the human superior parieto-occipital cortex encodes objects reachable by the hand. J Neurosci 29: 4381-4391, 2009.

Grosbras MH, Paus T. Transcranial magnetic stimulation of the human frontal eye field facilitates visual awareness. Eur J Neurosci 18: 3121-3126, 2003.

Gutteling TP, Van Ettinger-Veenstra HM, Kenemans JL, Neggers SF. Lateralized frontal eye field activity precedes occipital activity shortly before saccades: evidence for cortico-cortical feedback as a mechanism underlying covert attention shifts. J Cogn Neurosci 22: 1931-1943, 2010.

Gutteling TP, Kenemans JL, Neggers SF. Grasping preparation enhances orientation change detection. PLoS One 6: e17675, 2011.

Hommel B, Musseler J, Aschersleben G, Prinz W. The Theory of Event Coding (TEC): a framework for perception and action planning. Behav Brain Sci 24: 849-878, 2001.

Kammer T, Vorwerg M, Herrnberger B. Anisotropy in the visual cortex investigated by neuronavigated transcranial magnetic stimulation. Neuroimage 36: 313-321, 2007.

Lugo E, Doti R, Faubert J. Ubiquitous crossmodal Stochastic Resonance in humans: auditory noise facilitates tactile, visual and proprioceptive sensations. PLoS One 3: e2860, 2008.

Mills KR, Boniface SJ, Schubert M. Magnetic brain stimulation with a double coil: the importance of coil orientation. Electroencephalogr Clin Neurophysiol 85: 17-21, 1992.

Monaco S, Cavina-Pratesi C, Sedda A, Fattori P, Galletti C, Culham JC. Functional magnetic resonance adaptation reveals the involvement of the dorsomedial stream in hand orientation for grasping. J Neurophysiol 106: 2248-2263, 2011.

Moore T, Armstrong KM, Fallah M. Visuomotor origins of covert spatial attention. Neuron 40: 671-683, 2003.

Moore T, Fallah M. Microstimulation of the frontal eye field and its effects on covert spatial attention. J Neurophysiol 91: 152-162, 2004.

Moss F, Ward LM, Sannita WG. Stochastic resonance and sensory information processing: a tutorial and review of application. Clin Neurophysiol 115: 267-281, 2004.

Murata A, Gallese V, Luppino G, Kaseda M, Sakata H. Selectivity for the shape, size, and orientation of objects for grasping in neurons of monkey parietal area AIP. J Neurophysiol 83: 2580-2601, 2000.

Nakamura H, Kuroda T, Wakita M, Kusunoki M, Kato A, Mikami A, Sakata H, Itoh K. From three-dimensional space vision to prehensile hand movements: the lateral intraparietal area links the area V3A and the anterior intraparietal area in macaques. J Neurosci 21: 8174-8187, 2001.

Neggers SF, Bekkering H. Integration of visual and somatosensory target information in goal-directed eye and arm movements. Exp Brain Res 125: 97-107, 1999.

Neggers SF, Huijbers W, Vrijlandt CM, Vlaskamp BN, Schutter DJ, Kenemans JL. TMS pulses on the frontal eye fields break coupling between visuospatial attention and eye movements. J Neurophysiol 98: 2765-2778, 2007.

Oldfield RC. The assessment and analysis of handedness: the Edinburgh inventory. Neuropsychologia 9: 97-113, 1971.

Prablanc C, Pelisson D, Goodale MA. Visual control of reaching movements without vision of the limb. I. Role of retinal feedback of target position in guiding the hand. Exp Brain Res 62: 293-302, 1986.

Ruff CC, Bestmann S, Blankenburg F, Bjoertomt O, Josephs O, Weiskopf $\mathbf{N}$, Deichmann R, Driver J. Distinct causal influences of parietal versus frontal areas on human visual cortex: evidence from concurrent TMS-fMRI. Cereb Cortex 18: 817-827, 2008.

Ruff CC, Blankenburg F, Bjoertomt O, Bestmann S, Freeman E, Haynes JD, Rees G, Josephs O, Deichmann R, Driver J. Concurrent TMS-fMRI and psychophysics reveal frontal influences on human retinotopic visual cortex. Curr Biol 16: 1479-1488, 2006.

Sakata H, Taira M, Murata A, Mine S. Neural mechanisms of visual guidance of hand action in the parietal cortex of the monkey. Cereb Cortex 5: 429-438, 1995.

Sandrini M, Umiltà C, Rusconi E. The use of transcranial magnetic stimulation in cognitive neuroscience: a new synthesis of methodological issues. Neurosci Biobehav Rev 35: 516-536, 2011. 
Schutter DJ, Van Honk J. A standardized motor threshold estimation procedure for transcranial magnetic stimulation research. J ECT 22: 176-178, 2006.

Shikata E, Hamzei F, Glauche V, Knab R, Dettmers C, Weiller C, Büchel C. Surface orientation discrimination activates caudal and anterior intraparietal sulcus in humans: an event-related fMRI study. J Neurophysiol 85: 1309-1314, 2001.

Shikata E, Hamzei F, Glauche V, Koch M, Weiller C, Binkofski F, Büchel C. Functional properties and interaction of the anterior and posterior intraparietal areas in humans. Eur J Neurosci 17: 1105-1110, 2003.

Taira M, Mine S, Georgopoulos AP, Murata A, Sakata H. Parietal cortex neurons of the monkey related to the visual guidance of hand movement. Exp Brain Res 83: 29-36, 1990.

Taubert M, Dafotakis M, Sparing R, Eickhoff S, Leuchte S, Fink GR, Nowak DA. Inhibition of the anterior intraparietal area and the dorsal premotor cortex interfere with arbitrary visuo-motor mapping. Clin Neurophysiol 121: 408-413, 2010.

Tunik E, Frey SH, Grafton ST. Virtual lesions of the anterior intraparietal area disrupt goal-dependent on-line adjustments of grasp. Nat Neurosci 8: 505-511, 2005.

Van Elk M, Van Schie HT, Neggers SF, Bekkering H. Neural and temporal dynamics underlying visual selection for action. J Neurophysiol 104: 972 983, 2010.

Van Ettinger-Veenstra HM, Huijbers W, Gutteling TP, Vink M, Kenemans JL, Neggers SF. fMRI-guided TMS on cortical eye fields: the frontal but not intraparietal eye fields regulate the coupling between visuospatial attention and eye movements. J Neurophysiol 102: 3469-3480, 2009.

Wykowska A, Schubo A, Hommel B. How you move is what you see: action planning biases selection in visual search. J Exp Psychol Hum Percept Perform 35: 1755-1769, 2009.

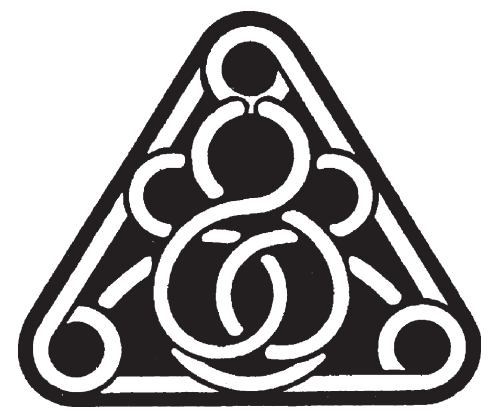

